\title{
Innovación docente en recursos humanos a través del aprendizaje-servicio. Una experiencia piloto
}

\author{
María Eugenia Sánchez Vidal \\ Yaiza Hernández García \\ Simón Hernández Aguado \\ Universidad Politécnica de Cartagena, España
}

\section{Resumen}

El artículo plantea la idoneidad de incluir los Objetivos de Desarrollo Sostenible (ODS) en el currículo académico de los estudiantes de Educación Superior. Para ello se justifica la idoneidad de la metodología de aprendizaje-servicio como metodología de aprendizaje activo, así como las ventajas y barreras de su implantación. Se expone la experiencia de una estudiante de grado de Administración y Dirección de Empresas que ha desarrollado su Trabajo Fin de Estudios mediante la metodología de aprendizaje-servicio. En concreto, el trabajo ha consistido en la realización de dos talleres formativos para estudiantes de Bachiller en Centros de Educación Secundaria sobre conciliación laboral y personal e igualdad de género. Los talleres se han realizado de manera exitosa en los centros de secundaria, y la estudiante ha conseguido la máxima calificación frente al tribunal. También se analiza la satisfacción y los comentarios realizados tanto por la estudiante como por los directores del Trabajo Fin de Estudios y los centros de secundaria receptores del servicio.

\section{Palabras clave}

Aprendizaje-servicio, recursos humanos, innovación docente, conciliación laboral y personal, objetivos de desarrollo sostenible. 


\title{
Teaching innovation in human resources through service- learning. A pilot experience
}

\begin{abstract}
This article analyses the benefits of embedding the Sustainable Development Goals (SDO) into the academic curriculum in Higher Education. To this end, we highlight the advantages of the service learning methodology and we relate a pilot experience involving a Business Administration and Management student working on a Human Resources Management project. The importance of service-learning as an active learning methodology is described, as well as the advantages and barriers of its implementation. This article relates the experience of a Business Administration and Management student who developed her Final Project through the service-learning methodology. Her project consisted in developing two formative workshops, aimed at secondary school students, on the topics of work and personal conciliation and on gender equality. The workshops were delivered successfully in secondary schools, and the student in question achieved the highest possible mark for her work. This paper also analyses satisfaction scores and comments made by the student, her project supervisors and staff at the secondary schools receiving the service.
\end{abstract}

\section{Keywords}

Service-learning, human resources, teaching innovation, work and personal conciliation, sustainable development goals. 


\section{Introducción}

La agenda para el desarrollo sostenible de Naciones Unidas establece 17 Objetivos de Desarrollo Sostenible (ODS) y 169 metas hacia cuya consecución trabajar hasta el año 2030. Las Universidades, como agentes educativos y de investigación, tienen también la obligación de asumir su responsabilidad hacia el logro de los ODS y debe ajustar sus prácticas docentes, investigadoras y de transferencia a la sociedad para alinearlas con dichas metas. Para ello es necesario que la docencia universitaria integre los ODS y utilice metodologías docentes que apuesten por la sostenibilidad del planeta y la educación en valores de sus estudiantes (UNESCO, 2009; Ministerio de Educación, 2010; CRUE, 2012).

Diferentes autores (Puig et al., 2007; Santos et al., 2015; Agrafojo et al., 2017) señalan que el aprendizajeservicio es una metodología idónea para construir un entorno natural, económico y social más sostenible, en línea con los ODS. El ApS es una propuesta educativa que persigue la solución de los problemas reales del entorno, a través de la acción donde los estudiantes prestan el servicio a una institución o asociación externa a su centro educativo para solucionar el problema o reto detectado.

Este trabajo aborda una experiencia de ApS en el área de Gestión de Recursos Humanos que se ha desarrollado a través del Trabajo Fin de Estudios (TFE) de una estudiante de Administración y
Dirección de Empresas. Concretamente, el servicio prestado por la estudiante ha consistido en la realización de dos talleres de Conciliación de la Vida Laboral y Personal en dos Centros de Educación Secundaria de Cartagena y Los Alcázares. El objetivo de los talleres ha sido llevar a un grupo de alumnos de entre 16 y 17 años -estudiantes de Economía de la Empresaconocimientos básicos sobre la conciliación y la igualdad de género, de manera que sean capaces de reconocer algunos de sus derechos, como futuros empleados, y el valor de la vida personal de los trabajadores, como potenciales miembros de la dirección de una empresa.

\section{Revisión de la literatura}

\subsection{El ApS como medio para contribuir a los ODS.}

Previa a la explicación de la metodología ApS es necesario definir qué se entiende por desarrollo sostenible y sostenibilidad. De acuerdo con el informe Brundtland (Comisión Mundial para el Medio Ambiente y el Desarrollo, 1987) el desarrollo sostenible se define como el desarrollo que permite satisfacer las necesidades de las generaciones presentes sin comprometer las necesidades de las generaciones futuras. En la actualidad, existen diversas y múltiples definiciones que enriquecen el debate sobre dicho concepto. Sin embargo, a pesar de esta diversidad, hay un consenso entre los distintos autores que relacionan al desarrollo sostenible con tres dimensiones principales (Artaraz, 2002): ambiental, social y 
económica. Por otro lado, en 2012, la Conferencia de Rectores de las Universidades Españolas (CRUE) definió la sostenibilidad como:

"un concepto que incluye la búsqueda de la calidad ambiental, la justicia social y una economía equitativa y viable a largo plazo. Define un conjunto de criterios orientados al comportamiento ético con todo lo que nos rodea (recursos, personas, espacios...), de modo que permita lograr una equidad intra e intergeneracional así como gestionar las relaciones con el medio natural y social, manteniendo su disponibilidad y equilibrio ecológico, y promoviendo una distribución más equitativa y justa de los recursos, beneficios y costes ambientales." (CRUE, 2012, p.7)

Este compromiso con el medioambiente, la economía y la justicia social ha sido consolidado a nivel mundial con la aprobación de la Agenda 2030 sobre el Desarrollo Sostenible por parte de los 193 miembros de las Naciones Unidas en el año 2015. En esta agenda se recogen 17 Objetivos de Desarrollo Sostenible (ODS) que incluyen desde la eliminación de la pobreza, hasta paliar el cambio climático, la educación de calidad, la igualdad de la mujer, la protección de los recursos naturales o la cooperación entre instituciones. Cada uno de estos objetivos está formado por distintas metas que deben lograrse para el año 2030, en total 169.

Para alcanzar estas metas, las universidades deben integrar la filosofía del desarrollo sostenible y de los ODS en su docencia. En este sentido, distintas instituciones (UNESCO, 2009; Ministerio de Educación, 2010; CRUE, 2012) indican la necesidad de incluir dicha filosofía en la educación universitaria para iniciar un cambio hacia la sostenibilidad en los valores y los criterios que orientan la decisiones de los estudiantes, pero también de los profesores, gestores y demás miembros de la comunidad universitaria.

En este sentido, la Educación para el Desarrollo Sostenible es un proceso de aprendizaje-enseñanza que promueve el cambio en los valores, expectativas, decisiones y modos de vida de la comunidad universitaria para orientarlos hacia la sostenibilidad (UNESCO, 2009). Esto implica desarrollar un conjunto de capacidades que no se pueden adquirir solo a través de la formación teórica

(Aramburuzabala et al., 2015). Objetivos como el cuidado de los ecosistemas terrestres, el consumo responsable, la solidaridad o la paz requieren que los alumnos se relacionen con su entorno social, económico y natural mediante un proceso de aprendizaje participativo donde las competencias, y también los valores, se enseñen y practiquen.

En esta línea es donde el ApS se considera hoy en día una metodología idónea para la Educación para el Desarrollo Sostenible. Una de las definiciones más aceptadas en la literatura sobre ApS establece que se trata de una propuesta educativa que combina procesos de aprendizaje y de servicio a la comunidad en un único 
proyecto bien articulado en el que los participantes aprenden a la vez que trabajan en necesidades reales del entorno con la finalidad de mejorarlo (Puig et al., 2007).

El ApS tiene dos componentes bien diferenciados. Por un lado el servicio que es el resultado o producto final que tiene que desarrollar un estudiante y que debe servir para resolver un problema del entorno o aportar alguna solución a un tema de interés general a través de una institución o agente social que lo solicita. Por otro lado, el aprendizaje que se asocia al servicio que debe desarrollar el estudiante bajo la guía-tutela del profesorado y que está vinculado al contenido curricular de una o varias asignaturas o al título que está cursando. En este sentido, cabe destacar, por tanto, las diferencias del ApS con la realización de prácticas (ya que no tienen porqué tener un componente social) ni con el voluntariado (que no necesariamente lleva asociado aprendizaje curricular ni seguimiento por parte de un académico) (Rodríguez-Gallego, 2013).

Desde el ApS se vislumbra una oportunidad para que los alumnos puedan abordar de una forma ética los problemas ambientales, sociales y económicos complejos, que afectan a la sostenibilidad del planeta (Martínez, 2008; Santos et al., 2015). Es decir, dicha metodología proporciona un medio ideal para lograr una Educación para el Desarrollo Sostenible para enriquecer el aprendizaje y promover el cambio social. Esta metodología se ha ido incrementando su presencia en los sistemas educativos hasta alcanzar una gran importancia hoy en día y estar presente en prácticamente en todo el mundo (Butin, 2006), con especial relevancia en algunos países de América Latina como México o Argentina (Barrios et al. 2011). En Europa, en cambio, esta metodología está en sus primeros pasos (Folgueiras et al., 2013), si bien en los últimos años se ha extendido su importancia y se espera que aumente más aún debido a la futura creación de un Observatorio Europeo de Aprendizaje Servicio (https://europeengage.org/). En relación a España, el ApS se ha introducido con más fuerza a partir del año 2000 con trabajos como los realizados por Martínez-Odría (2005) y gracias a grandes divulgadoras como es el caso de la pedagoga Roser Batlle (2011), perteneciente a la Asociación Española de Aprendizaje Servicio.

Entre las razones del auge de esta metodología se encuentran los beneficios que proporciona tanto a estudiantes como a docentes e instituciones y que se han ido identificando en el ámbito investigador por académicos como Astin, Vogelgesang, Ikeda y Yee, (2000) y Keen y Hall (2009) o Robinson y Torres (2007). Los investigadores señalan cómo el ApS ayuda a construir la identidad profesional de los estudiantes (Astin et al., 2000), desarrolla competencias personales, sociales y el autoconcepto (De la Cerda et al., 2008) al mismo tiempo que estimula la motivación de los estudiantes y el compromiso con su actividad (Martínez-Odría, 2006) y el pensamiento crítico (Sedlak et al. 2003). En paralelo se incrementa 
también la experiencia docente del profesorado, aumentando su satisfacción personal al promover una sociedad más junta a través de su contribución al logro de los ODS mediante su práctica docente (Bringle y Hatcher, 1996). Finalmente, la asociación o institución que solicita el servicio ve resuelta una necesidad o problema al ser los beneficiaros de la acción de ApS. En opinión de Tapia (2008) y Aramburuzabala et al. (2015) las oportunidades que proporciona el ApS pueden resumirse en los siguientes puntos siguiendo los estudios de investigaciones relevantes en este ámbito: 1) Diálogo y cultura de trabajo colectivo; 2) Establecimiento de vínculos entre la docencia, la misión de la Universidad y las necesidades de la comunidad; 3) Desarrollo de cambios en la estrategia didáctica que de más relevancia a las competencias trasversales y a una educación más experiencial y comprometida.

Por otro lado, los estudios de Aramburuzabala et al. (2015) muestran como el ApS es una innovación pedagógica que permite construir un futuro más sostenible, proporcionado un aprendizaje de calidad, responsable, interactivo, experiencial, transformador y relacionado con el mundo real. Para ello, se apoya en los principios básicos de sostenibilidad en el marco universitario aprobados por la CRUE en el año 2005, y revisados en el 2012. Los principios que se puede cumplir a través del ApS son:

- Principio ético. Mediante el ApS se puede analizar, debatir y desarrollar valores tales como la participación ciudadana, la solidaridad, el respeto a la diversidad o la igualdad mediante el aprendizaje. En este sentido, los docentes deben integrar estos valores en los contenidos, metodologías y sistemas de evaluación de las asignaturas, fomentando que los alumnos argumenten racionalmente su percepción de la realidad y se comprometan en la resolución de los problemas que afectan a la comunidad.

- Principio holístico. Los estudiantes estudian, cuestionan y abordan distintos temas desde una perspectiva holística e inclusiva. Los temas críticos para la comunidad requieren de una compresión desde distintas perspectivas para transformar la sociedad. Es decir, desde el ApS se utilizan enfoques éticos, ecológicos, sociales y económicos para abordar las problemáticas relativas al desarrollo sostenible.

- Principio de complejidad. El ApS permite trabajar con problemas reales y complejos, obteniendo una mejor comprensión de su origen, relación e impacto sobre el sistema social, ambiental, político, económico y cultural.

- Principio de globalización. El ApS ayuda a combinar los contenidos curriculares con acciones solidarias significativas tanto para los estudiantes como para la comunidad. En este sentido, los alumnos adquieren habilidades y conocimientos importantes para su futuro profesional y académico, mientras analizan, toman decisiones, resuelven, reflexionan y evalúan las necesidades de su entorno 
local más cercano, pero en el marco de una realidad global.

- Principio de transversalidad. Esta metodología podría ayudar a integrar contenidos de sostenibilidad en distintas asignaturas y titulaciones universitarias.

- Principio de Responsabilidad Social Universitaria. Mediante el ApS se puede afianzar el compromiso de las universidades con el desarrollo humano sostenible, mediante la colaboración con otras organizaciones sociales y educativas.

A pesar de la idoneidad del ApS para la Educación para el Desarrollo Sostenible y la importancia de avanzar hacia la integración del ApS en las universidades, este proceso implica una serie de dificultades que deben abordarse en el futuro (Jouannet et al., 2015). Estas dificultades incluyen 1) la concepción de la universidad como templo del saber que basa su docencia de manera predominante en la clase magistral; 2) la gran influencia que ejerce el mercado laboral en las enseñanzas universitarias condicionando contenidos en los planes de estudio y 3) la necesidad de realizar una clara articulación del aprendizaje en torno al servicio a ofrecer.

Afrontar estos retos no solo requiere incluir contenidos sobre sostenibilidad en las distintas asignaturas y titulaciones de las universidades, sino que es necesario realizar cambios institucionales que permitan convertir a la comunidad en un espacio donde se aprende, se investiga, se coopera y reflexiona. A pesar de los retos identificados, diferentes investigaciones (Martínez, 2008; Agrafojo et al., 2017) muestran como el aprendizaje servicio supone una oportunidad para avanzar hacia una nueva cultura y desarrollo institucional.

\subsection{El ApS en la Universidad Politécnica de Cartagena}

La Universidad Politécnica de Cartagena comienza su actividad de promoción del ApS en el año 2017 incorporando como eje formativo la formación del profesorado en valores y sostenibilidad, incluyendo de manera expresa la formación en temas de aprendizajeservicio. Este primer año se organiza desde el Vicerrectorado de Profesorado e Innovación Docente un curso de formación del profesorado a manos de la pedagoga Roser Batlle, perteneciente a la Asociación Española de Aprendizaje Servicio. A raíz del curso de formación realizado se derivan dos proyectos de innovación docente para el curso 20172018 y en el que están involucrados 20 profesores. Estos dos primeros proyectos han servido como proyectos promotores del ApS en la universidad.

El primero de los proyectos de innovación docente del curso 2017/18 se desarrolla en la Escuela Técnica Superior de Ingeniería Industrial y consiste en el desarrollo de un itinerario formativo para estudiantes del Grado de Ingeniería Industrial y Automática, con carácter voluntario, para el desarrollo de manos biónicas de bajo coste para la Fundación Rafa Puede, una asociación que persigue la mejora de la calidad de vida y de la autonomía de personas con 
discapacidad.

El segundo proyecto desarrollado durante el académico 2017/2018 ha perseguido el fomento y el impulso de trabajos de fin de estudios siguiendo la metodología ApS. Esto enfoque presenta varias ventajas frente a la incorporación de ApS en asignaturas concretas como, por ejemplo, la mayor flexibilidad de la temática a abordar en el servicio a ofrecer por los estudiantes, al no estar sujeto a un contenido curricular de una asignatura concreta. Se han desarrollado varios trabajos de fin de estudios dentro del proyecto de innovación docente como el desarrollo de una plataforma de gestión de un comedor escolar para un colegio público (Máster en Tecnologías de la Información y las Comunicaciones), el plan de visibilización de enfermedades raras (Grado en Administración y Dirección de Empresas) o un plan formativo para socios de una asociación de diabéticos de Cartagena (Grado en Administración y Dirección de Empresas).

En el curso académico 2018-2019 están en marcha 5 proyectos de innovación docente en los que están participando un total de 43 docentes, lo cual ha supuesto un notable incremento de participación del profesorado en esta iniciativa. Además de dar continuidad a los dos proyectos de innovación docente presentados en el curso académico anterior, es de destacar las nuevas iniciativas surgidas en la escuela Técnica Superior de Ingeniería Industrial y en la Escuela Técnica Superior de Ingeniería de Telecomunicación, así como en la
Escuela Técnica Superior de Arquitectura y Edificación y que abordan temas como el desarrollo de aplicaciones para la estimulación multisensorial en atención temprana, el fomento del emprendimiento a través del ApS o un proyecto arquitectónico para una asociación de vecinos de la ciudad de Murcia. Adicionalmente, a los proyectos de innovación en curso, actualmente se están desarrollando diversos trabajos de fin de estudios en la modalidad de ApS, demostrando el apoyo del profesorado a estas iniciativas docentes.

Finalmente, es de destacar la reciente creación de un grupo del fomento e implantación de los ODS en la universidad en noviembre de 2018, siendo uno de los ejes el fomento del ApS como medio para contribuir a dichos objetivos.

\section{Metodología}

3.1 Características generales del trabajo de fin de estudios

El trabajo de fin de estudios ha consistido en realizar dos talleres de Conciliación Laboral y Personal en centros de secundaria de Cartagena y Los Alcázares para estudiantes de $10 \mathrm{y}$ $2^{\circ}$ de Bachillerato. Para la selección de los centros de secundaria se han empleado los contactos personales de la estudiante y de los directores del trabajo. Una vez reunidos con los profesores de secundaria se identifica el reto de enriquecer las asignaturas de Economía de la Empresa con temas de conciliación laboral y personal y de igualdad de género y de mejorar el contacto y la relación de los Estudios de

Sánchez, M.E.; Hernández, Y. y Hernández, S. (2019). Innovación docente en recursos humanos a través del aprendizaje-servicio. Una experiencia piloto. RIDAS, Revista Iberoamericana de Aprendizaje Servicio, 7, 1-16. DOI10.1344/RIDAS2019.7.1 
Secundaria con los estudios de Educación Superior. El servicio consiste, pues, en realizar unos talleres en los centros de Secundaria y el aprendizaje, asociado a la experiencia de la estudiante, está relacionado con asignaturas de dirección de Recursos Humanos de las empresas, con especial hincapié en prácticas de conciliación laboral y personal e igualdad de género. Además de aumentar sus conocimientos sobre estos temas se potencian competencias personales y valores de la estudiante, siendo las más destacadas la comunicación oral y expositiva al tener que poner en uso estas habilidades frente una audiencia y el desarrollo de un mayor compromiso y sensibilidad hacia el tema de la conciliación laboral y personal igualdad de género.

Con la realización de este trabajo se ha buscado trabajar y sensibilizar en torno a los 4 de los 17 ODS de Naciones Unidas, en concreto con el ODS 4 (Educación de calidad) pues propone profundizar y ampliar conocimientos sobre prácticas de recursos humanos de la estudiante de Administración y Dirección de Empresas y de los estudiantes de Bachillerato que con manera frecuente no se pueden abordar en asignaturas básicas de sus estudios oficiales; el ODS 5 (Igualdad de Género) pues aumenta la sensibilidad hacia el tema de la desigualdad de género entre los estudiantes de Bachillerato al tratar temas como la conciliación, las diferencias de género en el conflicto de roles, la corresponsabilidad y la igualdad de oportunidades en el trabajo; el ODS 8 (Trabajo decente y crecimiento económico) pues se analizan las medidas de conciliación como un medio para obtener empleos de calidad al mismo tiempo que trabajadores comprometidos y empresas más productivas y finalmente, el ODS 10 (Reducción de las desigualdades) pues el taller persigue transmitir a los estudiantes de Bachillerato que la conciliación debe romper con los estereotipos establecidos socialmente y que las medidas de conciliación en las empresas no deben ser orientadas en exclusividad a mujeres que trabajan y tienen hijos, sino a aquellas personas que buscan equilibrar su vida laboral y su vida personal, independientemente del género y de tener o no tener descendencia. Finalmente, con este trabajo se pretende establecer alianzas de colaboración, fundamentales también en el marco de los ODS con el ODS 17 (alianzas para lograr los objetivos).

3.2 Objetivos de aprendizaje del taller y herramientas didácticas a utilizar

El trabajo de fin de estudios se ha desarrollado en dos etapas claramente diferenciadas. La primera de ellas ha consistido en el desarrollo del marco conceptual que ha dado lugar al desarrollo teórico del trabajo mediante el cual la estudiante ha profundizado en los conceptos que posteriormente debía explicar a los estudiantes de

Secundaria. Los temas abordados en la primera parte del trabajo incluyen la conciliación laboral y personal, el conflicto entre roles, las medidas de conciliación laboral y personal y la situación de la conciliación y de la 
igualdad en España.

La segunda parte del trabajo ha consistido en la realización de los talleres en los centros de secundaria, así como en la redacción de la experiencia y en realizar una reflexión sobre la experiencia vivida. Para la planificación de los talleres ha sido necesario establecer claramente cuáles eran los objetivos de aprendizaje de los estudiantes de Bachillerato, teniendo en cuenta el tiempo de que se dispone para su realización. Se establecen como prioritarios varios objetivos. Primero, dar significado al concepto de conciliación de manera que los estudiantes comprendan de qué se trata exactamente ya que es un concepto de creciente popularidad en la sociedad cuyo significado no siempre está claro. Junto con este concepto, se establecen como objetivo explicar en qué consiste el conflicto entre roles, las consecuencias de la falta de conciliación, las diferencias de género en el conflicto de roles y la importancia de la corresponsabilidad en el ámbito doméstico. Finalmente, se pretende que los estudiantes conozcan las soluciones que frecuentemente se ofrecen en las empresas para la mejorar la conciliación, así como los efectos positivos de la utilización de dichas prácticas para empleados y organizaciones.

En cuanto a las herramientas didácticas a emplear en los talleres, se plantea una presentación muy visual, con gran contenido de imágenes y videos para conseguir atraer y captar la atención de los adolescentes. Además de lo anterior, se planifica la realización de una actividad de gamificación que, según la literatura, resulta una herramienta muy atractiva para lograr el compromiso y la motivación de los estudiantes (Sánchez, 2015; Caraballo et al., 2017). En concreto, un concurso al final del taller a través de un juego de preguntas y respuestas a través del teléfono móvil u otro dispositivo, en el que los estudiantes puedan demostrar si realmente han entendido y asimilado los conceptos que se han mostrado en la presentación previa. Para la realización del juego, se decide utilizar la aplicación Kahoot, una plataforma gratuita y divertida que permite una mejor interacción con los alumnos y que está adquiriendo una gran popularidad.

Para medir el aprendizaje de esta experiencia piloto se han utilizado distintas variables cuantitativas. Por un lado, el aprendizaje de la autora del trabajo de fin de estudios medido a través de su calificación frente al tribunal de la defensa en la Facultad de la Empresa y, por otro, los resultados de aprendizaje de los estudiantes de Bachillerato medidos a través de los resultados obtenidos en el juego de competición Kahoot. Por otro lado, se recogen mediante observación directa y entrevistas informales una valoración del impacto del taller más cualitativa que incluye impresiones y comentarios recibidos o realizados por la autora del trabajo, los directores del trabajo, así como el profesorado de los centros de Secundaria donde se han realizado los talleres. 


\section{Desarrollo de las experiencias}

\subsection{Experiencia Colegio Las Claras del Mar Menor.}

El desarrollo del taller se llevó a cabo en el centro educativo concertado Las Claras del Mar Menor, con estudiantes de entre 16 y 17 años del grado $1^{\circ}$ de Bachillerato de Ciencias Sociales. La propuesta se realizó para dos sesiones de 50 minutos aproximadamente cada una. El contenido de cada sesión se dispuso de la siguiente manera: por un lado, la primera sesión contendría la presentación sobre conciliación laboral y personal, por otro lado, la segunda sesión se dividiría en un leve repaso de lo visto en la sesión anterior, la elaboración de un juego interactivo con preguntas tipo test sobre la presentación. Por último, un coloquio o tiempo para preguntas, en el que los alumnos podrían comentar sus opiniones o realizar preguntas sobre cuestiones que no habían quedado claras.

Cabe decir que, a pesar de esta planificación, la participación a lo largo de la presentación fue tan numerosa, que resultó imposible terminar la presentación en una sesión. De tal forma, se continuó la presentación en la segunda sesión y hubo que eliminar la parte de juego por falta de tiempo, pero también porque el centro tiene prohibido por normativa el uso del móvil u otros dispositivos en el aula.

Finalmente, destacar que la experiencia resultó notablemente positiva: Ios alumnos entendieron los conceptos y mostraron su interés por el taller y por el tema que se estaba tratando.
Además, su alta participación (con la que no se contaba), aunque accidentó la planificación, resultó muy positiva, ya que favoreció la discusión y el debate, mantuvo la expectación y el interés entre la audiencia.

\subsection{Experiencia I.E.S. Mediterráneo}

En este caso, el taller se realizó en el centro I.E.S. Mediterráneo de

Cartagena con dos grupos de alumnos y en sesiones separadas: por un lado, una primera presentación con alumnos del grado $1^{\circ}$ Bachillerato de Ciencias Sociales, y por otro lado, se realizó una segunda ronda con los alumnos del grado $2^{\circ}$ Bachillerato de Ciencias Sociales. Analizadas las claves de éxito y los errores cometidos en la experiencia anterior, se realizaron ciertas modificaciones en el planteamiento del taller en relación a los contenidos y a la gestión del tiempo. Así, en lugar de dos sesiones para cada grupo de 50 minutos cada una, se acortó el tiempo, de tal manera que, cada grupo de alumnos recibiría una única sesión para la presentación de contenidos y la realización del juego final de preguntas y respuestas. La organización temporal del taller fue la siguiente: presentación de los conceptos y de la temática del taller (35 minutos) y juego concurso mediante la aplicación kahoot (15 minutos). Para premiar a los ganadores de esta actividad la Facultad de Ciencias de la Empresa donó unos premios consistentes en unas bolsasmochilas con una camiseta de la Facultad y otros obsequios institucionales. El desarrollo de las sesiones fue muy positivo y se cumplió

Sánchez, M.E.; Hernández, Y. y Hernández, S. (2019). Innovación docente en recursos humanos a través del aprendizaje-servicio. Una experiencia piloto. RIDAS, Revista Iberoamericana de Aprendizaje Servicio, 7, 1-16. DOI10.1344/RIDAS2019.7.1 
con la planificación establecida ajustada al tiempo disponible. El público en cada caso fue distinto, el primer grupo fue receptivo y permitió una mayor interacción tanto en la presentación como en el juego realizado, mientras que, con el segundo grupo, resultó más complicado romper la barrera y permitir una participación activa. No obstante, se observó mejor compresión de los conceptos e ideas en este segundo grupo.

\section{Resultados}

Como se ha explicado, los resultados de la experiencia de ApS se pueden valorar desde distintos puntos de vista. En primer lugar, en el ámbito puramente académico y en relación al aprendizaje, el tribunal que valoró el Trabajo final de estudios le otorgó a la estudiante la calificación de Sobresaliente y propuso el trabajo para recibir la mención de Matrícula de Honor. Cabe destacar que la normativa académica vigente establece que una comisión fijará los trabajos final de estudios merecedores de la distinción y, así pues, se trata de un proceso de calificación competitiva, en el que se valora el trabajo en relación al resto de trabajos presentados en el mismo curso académico en la Facultad de Ciencias de la Empresa.

En cuanto al aprendizaje en los estudiantes de Secundaria, se han valorado las respuestas que dieron en las preguntas del cuestionario final del taller. Cabe recordar que en estos cuestionarios se preguntaba sobre conceptos básicos explicados en el taller en torno a corresponsabilidad, conflicto de roles, conciliación laboral y personal, medidas de conciliación e igualdad de género. En este caso, solo se ha podido valorar el aprendizaje de los estudiantes de los dos talleres realizados en el Centro IES Mediterráneo, ya que en la primera experiencia no se puedo realizar el concurso debido a los motivos anteriormente expuestos. Los resultados han sido moderadamente satisfactorios, si bien, existen diferencias importantes de aprendizaje entre un grupo y otro, aspecto que había sido detectado intuitivamente en el Trabajo final de estudios. Un análisis individualizado de las preguntas permite detectar las preguntas que tienen un menor porcentaje de aciertos, información que podrá ser de utilidad para aclarar conceptos en un futuro taller, o bien, para reformular el enunciado de las preguntas realizadas.

En relación a la satisfacción, impresiones y comentarios recibidos por los centros receptores del servicio caben destacar varios aspectos. Como se ha comentado, la experiencia en Colegio Las Claras del Mar Menor resultó altamente positiva a nivel de participación del alumnado e interés mostrado por la audiencia. Además de lo anterior, tanto el centro como los propios alumnos felicitaron a la estudiante por el taller realizado y obtuvieron sensaciones muy positivas al respecto, siendo elegida como una de las actividades mejor valoradas del curso académico 2017-18 en las encuestas de satisfacción que se realizan anualmente en el Centro Educativo. Por otro lado, en el IES Mediterráneo el profesorado del centro 
implicado comunicó el agradecimiento y la satisfacción con la actividad, dejando abierta la posibilidad de realizar el taller para otros cursos o para otras colaboraciones.

Finalmente, se han recogido las impresiones de la estudiante encargada de realizar los talleres, así como las impresiones de la directora y codirector del trabajo mediante un video ${ }^{i}$ para el fomento del AprendizajeServicio en la Universidad Politécnica de Cartagena. La estudiante manifiesta un gran sensación de logro frente al reto que le supuso la realización de los talleres y afirma que la experiencia "le ha hecho muy feliz", le ha resultado una experiencia muy enriquecedora y muy positiva a nivel personal. En la misma línea se manifiestan los directores del trabajo que señalan la gran satisfacción personal que les ha generado este proyecto, al ver cómo una estudiante es capaz de trasmitir unos conocimientos que han sido previamente adquiridos a través de ellos en la Facultad de Ciencias de la Empresa y hacerlo de manera tan profesional. Además, destacan la importancia de educar en valores a los estudiantes de Administración y Dirección de Empresas como medio para el logro de un mundo más sostenible, en torno a los ODS.

\section{Conclusiones}

Este artículo pone de manifiesto el poder de transformación social del ApS, al mismo tiempo que ofrece a las Universidades una vía para poder implantar y apostar por el compromiso social esencial dentro de la
Responsabilidad Social Corporativa y contribuir a los ODS (Agrafojo et al., 2017). El ApS constituye, además, una vía para aumentar la calidad docente ofrecida al estudiantado mediante oportunidades de aprendizaje real y útil y el desarrollo de competencias y valores asociados a la creación de una ciudadanía participativa y transformadora.

En el artículo se muestra una iniciativa piloto de ApS en la Facultad de Ciencias de la Empresa que consiste en llevar dos talleres de conciliación laboral y personal a centros de secundaria a través de la realización del TFE de una estudiante de grado de Administración y Dirección de Empresas. Los resultados de aprendizaje ofrecen datos muy satisfactorios para la estudiante encargada de la realización de los talleres y moderadamente satisfactorios en relación al aprendizaje de los estudiantes de Bachillerato. Sin embargo, resulta casi más interesante analizar las impresiones y comentarios recibidos en torno a la experiencia que ponen de manifiesto el poder motivador de esta iniciativa docente tanto para la estudiante como los docentes participantes en la experiencia.

A pesar de lo anterior, conviene mostrar las limitaciones del trabajo que se ha presentado. Al ser un proyecto innovador y piloto en la Facultad de Ciencias de la Empresa no se planteó inicialmente como artículo de investigación y esto ha impedido establecer un protocolo para medir el impacto del taller, por ejemplo, mediante cuestionarios a docentes y estudiantes de los centros de 
secundaria. Tampoco ha existido una valoración del desarrollo de competencias personales o del poder transformador de la experiencia en la estudiante que lo ha llevado a cabo. Estas limitaciones podrán ser tenidas en consideración en futuros trabajos de ApS que se desarrollen.

Finalmente, nos gustaría destacar las principales contribuciones que este trabajo realiza. En primer lugar, ha sido la primera experiencia de ApS realizada en la Facultad de Ciencias de la Empresa y una de las primeras realizadas en la UPCT. El éxito en el desarrollo de la experiencia permite dar valor a una metodología de aprendizaje activo comprometida socialmente con el logro de los ODS, algo todavía novedoso en el panorama universitario. El vídeo de la experiencia se ha colgado en la web de innovación docente de la UPCT para la difusión y fomento de la metodología entre el profesorado universitario. Asimismo, este trabajo puede servir de referencia y guía para docentes de asignaturas del área de Organización de Empresas y concretamente, de Gestión de Recursos Humanos, ya que no se han encontrado experiencias previas en este ámbito en la literatura académica.

\section{Referencias bibliográficas}

Association for the Advancement of Sustainability in Higher Education. (2010). Sustainability curriculum in higher education: A call to action. Denver, Colorado: Association for the Advancement of Sustainability in Higher Education.

Agrafojo, J., García, B., y Jato, E.
(2017). Aprendizaje servicio e innovación educativa en la Universidad de Santiago de Compostela: estrategia para su institucionalización. RIDAS. Revista Iberoamericana de Aprendizaje Servicio, 3, 23-34.

Aramburuzabala, P., Cerrillo, R., y Tello, I. (2015). Aprendizaje-servicio: una propuesta metodológica para la introducción de la sostenibilidad curricular en la universidad. Profesorado. Revista de Currículum y Formación de Profesorado, 19(1), 7895.

Astin, A. W., Vogelgesang, L. J., Ikeda, E. K., y Yee, J. A. (2000). Executive summary: How service learning affects students. Los Angeles: Higher Education Research Institute, UCLA.

Barrios Araya, S., Rubio Acuña, M., Núñez, M. G., y Vería, C. S. (2011). Aprendizaje-servicio como metodología para el desarrollo del pensamiento crítico en educación superior. Revista Cubana de Educación Médica Superior, 26(4), 594-603.

Batlle, R. (2011). ¿ De qué hablamos cuando hablamos de aprendizajeservicio. Crítica, 972(61), 49-54.

Bringle, R. G., y Hatcher, J. A. (1996). Implementing service learning in higher education. The Journal of Higher Education, 67(2), 221-239.

Butin, D. E. (2006). Future Directions for Service Learning in Higher Education. International Journal of Teaching and Learning in Higher Education, 18(1), 1-4. 
Caraballo, A. M. M., Peinado, P. H., y González, M. M. S. (2017).

Gamificación en la educación, una aplicación práctica con la plataforma Kahoot. Anales de ASEPUMA, 25, 2-13. De la Cerda, M., Martín, X., y Puig, J. M. (2008). Amigos y amigas de lectura. Una experiencia de aprendizaje servicio en la formación de profesionales de la educacióm. En M. Martínez (Coord.). Aprendizaje Servicio y Responsabilidad Social de las universidades (pp. 129150). Barcelona: Octaedro.

Conferencia de Rectores de las Universidades Españolas. (2012). Directrices para la introducción de la sostenibilidad en el curriculum. Recuperado de https://www.crue.org/Documentos\%20 compartidos/Declaraciones/Directrices Sosteniblidad Crue2012.pdf

Conferencia de Rectores de las Universidades Españolas. (2015). Institucionalización del AprendizajeServicio como estrategia docente dentro del marco de la Responsabilidad Social Universitaria para la promoción de la Sostenibilidad en la Universidad. Recuperado de https://www.crue.org/Documentos $\% 20$ compartidos/Recomendaciones $\% 20 y \%$ 20criterios\%20tecnicos/2.\%20APROBA DA\%20INSTITUCIONALIZACION\%20Ap S.pdf

Folgueiras, P., Luna, E., y Puig, G. (2013). Aprendizaje y servicio: estudio del grado de satisfacción de estudiantes universitarios. Revista de Educación, 362, 159-185.

Keen, C., y Hall, K. (2009). Engaging with difference matters: Longitudinal student outcomes of co-curricular service-learning programs. The Journal of Higher Education, 80(1), 59-79.

Martínez, M. (2008). Aprendizaje servicio y responsabilidad social de las universidades. Barcelona: OctaedroICE.

Ministerio de Educación (2010).

Estrategia Universidad 2015.

Recuperado de

http://www.educacion.gob.es/eu2015/l a-eu2015.html

Puig, J. M., Batlle, R., Bosch, C., y Palos, J. (2007). Aprendizaje servicio. Educar para la ciudadanía. Barcelona: Octaedro.

Rodríguez Gallego, M. R. (2014). El Aprendizaje-Servicio como estrategia metodológica en la Universidad. Revista Complutense de Educación, 25(1), 95113.

Santos, M. Á..., Sotelino, A., y Lorenzo, M. (2015). Aprendizaje-servicio y misión cívica en la universidad: Una propuesta de desarrollo. Barcelona: Octaedro.

Sedlak, C. A., Doheny, M. O., Panthofer, N., y Anaya, E. (2003). Critical thinking in students' service-learning experiences. College Teaching, 51(3), 99-104.

Tapia, M. N. (2008). Calidad académica y responsabilidad social: el aprendizaje servicio como puente entre dos culturas universitarias. Aprendizaje servicio y responsabilidad social de las universidades, 27-56. 
UNESCO. (2009). Educación Superior y Objetivos de Desarrollo Sostenible.

Recuperado de

https://es.unesco.org/themes/educacio

n-superior/ods

${ }^{\mathrm{i}}$ https://media.upct.es/videos/?vim =MzE5Ng 